\title{
Insights into cancer mechanisms from genomic research on urological cancers
}

\author{
Wolfgang A Schulz* and Teodora Ribarska
}

\begin{abstract}
Molecular mechanisms driving cancer development and progression are rarely unique to one cancer type. Rather, recent genomic studies of urological cancers suggest that common mechanisms recur with variations. Examples include alterations in hypoxia response regulation, epigenetic regulator proteins, and signal transduction pathways in renal, prostatic and urothelial carcinomas. Consideration of these variations alongside the common basic cancer mechanisms might be important for the successful development of targeted therapies.
\end{abstract}

\section{Introduction}

Over the past decades numerous common molecular mechanisms underlying the development of human cancers have been identified. It now seems that each subtype of human cancer is driven by a specific assortment of selected cancer mechanisms. Notably, in various cancers the same basic mechanisms act in different ways and to different degrees. A theme identified in one cancer often turns up as a reprise with variations in others.

For instance, overactivation of the canonical Wnt signaling pathway is crucial to the development of many cancers in the gastrointestinal tract. In colorectal cancers constitutive pathway activity is caused predominantly by inactivation of its negative regulator APC, whereas the typical alteration in gastric and hepatocellular cancers is mutational activation of the central signal transducer $\beta$ catenin. In genitourinary cancers, Wnt pathway activation is more subtle. In cancers of the kidney, bladder or prostate, mutations in intracellular Wnt pathway components are rare and instead, epigenetic silencing of SFRP, $D K K$ and WIF1 genes encoding extracellular Wnt antagonists is prevalent (Figure 1a). Presumably, these changes enhance the effect of paracrine or autocrine Wnt growth

*Correspondence: wolfgang.schulz@uni-duesseldorf.de Department of Urology, Heinrich Heine University, Düsseldorf, Germany factors but do not lead to a constitutively active state of the pathway [1]. Why these differences exist is an intriguing question for cancer researchers and developmental biologists.

In the past, many cancer mechanisms - such as the pivotal role of Wnt signaling in colorectal cancer - were discovered by elucidating the function of single genes, often in the course of studying rare inherited cancer syndromes. Large-scale genomic techniques have opened up new ways to investigate cancer mechanisms, especially in cancer types that are not associated with inherited syndromes, which include the common cancers of the prostate and the urinary bladder. Over the past decade, array techniques for gene expression profiling and detection of gene copy number changes have been instrumental. More recently, high-throughput sequencing techniques have begun to contribute important insights by allowing comprehensive mutation detection or identification of in vivo transcription factor binding sites following chromatin immunoprecipitation (ChIP).

\section{Renal cancer: aberrant hypoxia regulation as a primary mechanism in carcinogenesis}

Investigations of clear-cell renal cell carcinoma (ccRCC), the major histological subtype of renal carcinoma, have highlighted the importance of hypoxia in cancer generally. Recent large-scale sequencing studies on ccRCC identified mutations predominantly in genes involved in the cellular response to hypoxia, including several chromatin modifying or remodeling proteins $[2,3]$. The most common genetic alterations in ccRCC lead to inactivation of the $V H L$ tumor suppressor gene, which is mutated in the germline of patients suffering from the hereditary vonHippel-Lindau (VHL) tumor syndrome. A major consequence of $V H L$ functional loss is permanent activation of the hypoxia-induced factors HIF $2 \alpha$ or HIF1 $\alpha$ [4]. These transcription factors direct changes in cellular metabolism, enhance angiogenesis, activate autocrine growth factor circuits, and induce proapoptotic proteins in response to low oxygen. HIF1 $\alpha$ is also induced in many other human cancers, allowing growth and survival under hypoxic conditions and promoting angiogenesis, but VHL inactivation is uncommon. 


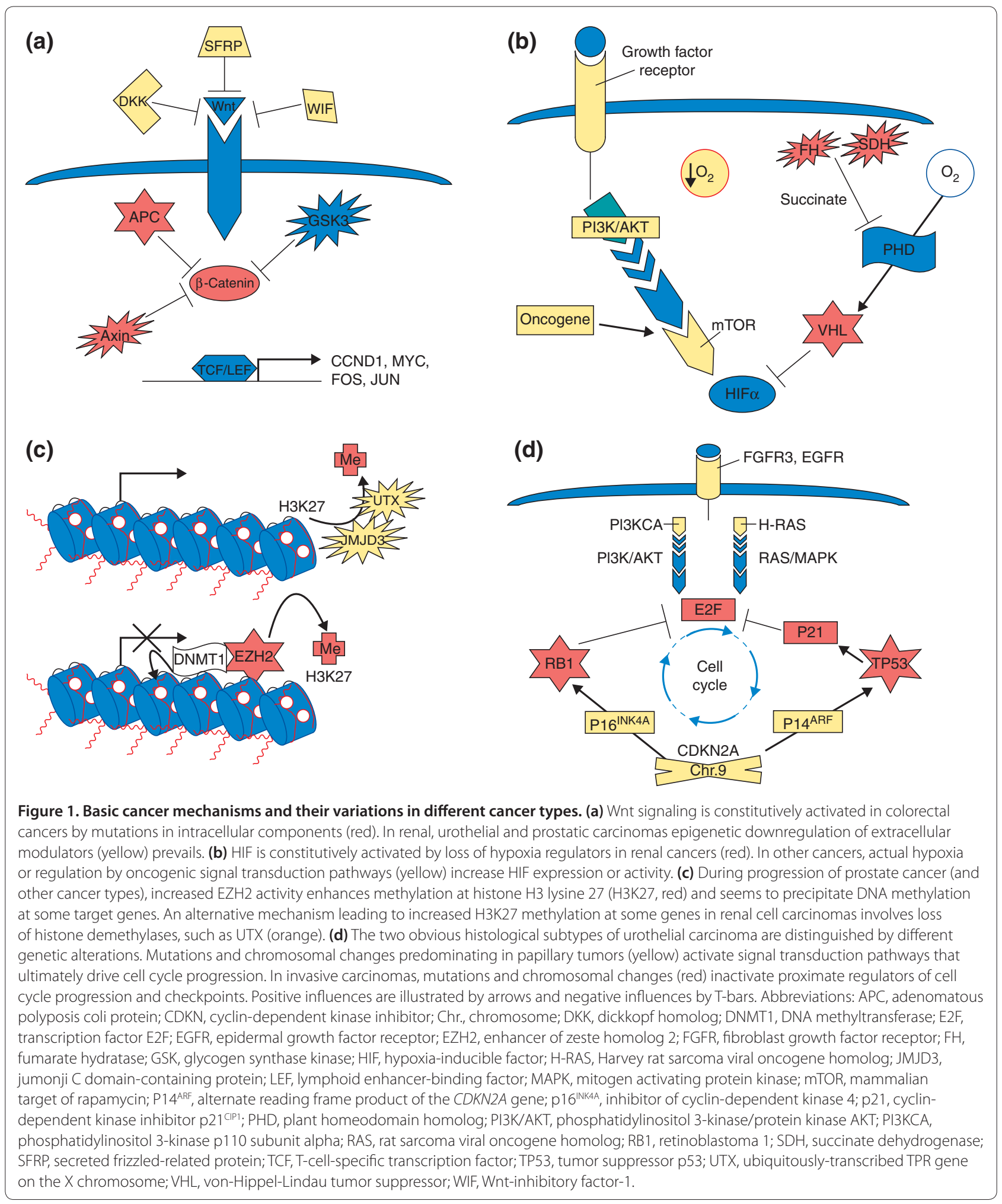

Nevertheless, there are significant differences in the details of how hypoxic regulation is disturbed between ccRCC and other cancers (Figure 1b). The activation of hypoxia responses by loss of VHL function is essentially unique to ccRCC and represents an early initiating event in cancer development. Completely independent of actual oxygen availability, these cancers execute a program for the cellular response to hypoxia - achieving a 
state consequently termed 'pseudohypoxia'. Pseudohypoxia may lead to cancer development when the autocrine growth factor circuits it induces are stabilized while the concomitantly induced proapoptotic genes are silenced.

Intriguingly, a number of rare hereditary cancer syndromes caused by mutations in unlikely tumor suppressors, such as fumarate hydratase and succinate dehydrogenase, show a similarly remarkable preference for causing tumors in the kidney, albeit not necessarily ccRCC [4]. In these cancers, interruption of the tricarboxylic acid cycle results in elevated levels of fumarate or succinate, leading to inhibition of the proline and asparagine hydroxylases that prevent HIF stabilization and activation. By comparison, in other cancers, adaptation to hypoxia takes place during tumor progression when HIF $1 \alpha$ is activated as a downstream consequence of oncogenic pathways. In many cases, these act via mammalian target of rapamycin (mTOR), a regulator of mRNA translation and cell growth. Drugs targeting aberrant HIF activation have been introduced into the clinic and have indeed proven efficacious, most of all in the treatment of metastatic ccRCC. They may be particularly successful in this cancer because it depends uniquely on this mechanism, not only for angiogenesis. In addition, the particular HIF activated in ccRCC is often HIF2 $\alpha$, which has a different spectrum of target genes from HIF1 $\alpha$ [4]. Thus, constitutive activation of the hypoxic response seems to be a crucial primary mechanism in renal carcinogenesis. It is tempting to speculate that the mutations in chromatin modifying and remodeling proteins recently identified in $\mathrm{RCC}[2,3]$ may serve to stabilize this aberrant state.

\section{Prostate cancer: histone modifying enzymes as oncogenes}

The histone methyltransferase EZH2 has emerged as a major driver of prostate cancer carcinogenesis. A recent genome-wide ChIP study revealed that it interacts with the androgen receptor and the ETS family transcription factor ERG to establish the aberrant differentiation state of prostate cancers [5]. EZH2 and ERG had both been identified as overexpressed in prostate cancer by gene expression microarrays. This technique has not only helped to elucidate novel mechanisms of prostate cancer development and progression, but has also provided biomarkers, such as the peroxisomal protein AMACR, which is now widely used to resolve ambiguous histological findings in prostate biopsies [6].

EZH2 is the catalytic subunit of the Polycomb complex PRC2 and catalyzes trimethylation of histone $\mathrm{H} 3$ on Lys27 (HeK27). This histone modification is typically associated with gene repression, as is the case for DNA hypermethylation of CpG-island promoters. Indeed, these two events were subsequently found to be related
(Figure 1c). In normal tissues, a subset of genes is marked by $\mathrm{H} 3 \mathrm{~K} 27 \mathrm{me} 3$ and bound by PRC2. These genes are normally weakly expressed or silent, but remain poised for activation. During carcinogenesis, they become fully silenced by DNA hypermethylation, often losing EZH2 occupancy and the H3K27me3 modification in the process. At some silenced genes, EZH2 and H3K27me3 may directly attract DNA methyltransferases, whereas at others, DNA hypermethylation occurs independently of Polycomb proteins. Conversely, some EZH2 target genes remain repressed in cancers without becoming (DNA-) hypermethylated [7].

Meanwhile, overexpression of EZH2 and other polycomb proteins, especially BMI1, has been observed in a range of other common and rare cancers. The specific histone modification established by EZH2 is reversed by the histone demethylases UTX and JMJD3. Intriguingly, UTX is inactivated by mutations in renal carcinomas [2]. These findings reveal a novel cancer mechanism, in which aberrant activity of epigenetic regulatory repressor proteins or inactivation of their antagonists drives cancer progression and facilitates DNA hypermethylation. They open a wealth of opportunities for diagnostic and therapeutic approaches in urological and other cancers.

\section{Urothelial cancers: distinguishing two routes to cancer}

Urothelial carcinoma, the major histological type of bladder cancer and the fifth most common noncutaneous cancer in industrialized countries, manifests as one of two subtypes, papillary tumors and more aggressive invasive cancers. Invasive cancers arise either from a flat high-grade dysplasia, from carcinoma in situ, or by progression from papillary tumors. Papillary cancers have near-diploid genomes, whereas invasive carcinomas are characterized by marked chromosomal instability [8]. Recent studies integrating global analyses of gene expression and genetic alterations clarify the different mechanisms acting in these subtypes [9].

Papillary tumors have a characteristic set of genetic changes: mutations activating the fibroblast growth factor receptor FGFR3, signal-transducing RAS proteins (most often HRAS) or the catalytic subunit of phosphatidylinositol 3-kinase PIK3CA. In addition, loss of chromosome 9 is frequent, targeting CDKN2A at 9p21 (encoding the cell cycle regulator $\mathrm{p} 16^{\mathrm{INK} 4 \mathrm{~A}}$ and the activator of $\mathrm{p} 53$ p14 ${ }^{\mathrm{ARF}}$ ) and various genes on 9q, such as TSC1 (an inhibitor of mTOR). Normal urothelial cells proliferate rapidly but transiently after tissue damage or in culture, under the influence of autocrine and paracrine factors of the epidermal growth factor (EGF) and FGF family, which in turn act through the mitogen activated protein kinase and phosphatidylinositol 3-kinase signal transduction pathways. The characteristic genetic changes in papillary 
urothelial tumors seem to result in overactivity of these same pathways, leading to continuous growth.

Except for chromosome 9 loss, the typical mutations in papillary tumors are less common in invasive urothelial cancers, which are instead often characterized by inactivation of the p53 and retinoblastoma protein (RB1) cellular control systems through various mechanisms (Figure 1d), alongside many other changes. Evidently, the inactivation of the p53 and RB1 systems compromises the function of cell cycle checkpoints responding to telomere erosion, chromosomal instability, and oncogeneinduced irregular replication. In addition, checkpoint signaling through ATR and CHK protein kinases is also suppressed in these cancers. Together, these changes may account for the limited effect of cytotoxic chemotherapy in the treatment of invasive bladder cancers. They could also be very pertinent to therapies using novel drugs directed at signal transduction pathways. Loss of the p53 and pRB1 control systems may uncouple proliferation of the tumor cells from extracellular signals, diminishing the requirement for signaling through these pathways. Tellingly, signatures of the overactive transcription factors E2F1 or E2F3, which are normally restrained by RB1 from driving cell proliferation and replication, dominate the expression profiles of invasive bladder cancers and distinguish them from papillary tumors [10].

The assortment of genetic changes observed in invasive urothelial carcinomas is similar to that encountered in other carcinomas, for example lung cancers and squamous cell carcinomas. With the expected variations, the theme emerging from the comparison of papillary and invasive bladder cancers might therefore be relevant in the pathogenesis of these cancers as well. For instance, the limited efficacy of small-molecule EGF receptor inhibitors in lung cancers and metastatic colorectal carcinomas may be caused not only by downstream mutations in signal transduction pathways, but also by an analogous uncoupling of cell cycle progression and cell proliferation from pathways driven by growth factor receptors by $\mathrm{p} 53$ mutations and RB1 inactivation.

\section{Conclusions}

Basic mechanisms driving cancer development frequently recur in different cancer types, but more often than not with variations that have ramifications that extend beyond heuristics to the design of novel therapeutic approaches. Many new questions follow from the insights sketched above. For instance, which mechanisms cause the constitutive activation of the hypoxic response in ccRCC cases lacking VHL mutations? Are these more similar to those mediating adaptation to hypoxia in other cancer types? Which targets of the epigenetic repression elicited by EZH2 deregulation in prostate cancers (and others) are crucial for pathogenesis and accessible to therapeutic approaches? How can urothelial (and other) cancers with overactive signal transduction pathways be reliably distinguished from those with failure of the central p53 and RB1 control systems in clinical practice, and which molecular targets for therapy can be chosen in cancers largely independent of extracellular signaling pathways? The techniques for genomic analyses that have been available in the past decade have helped to define these questions and the new generation of techniques should help to answer them, hopefully soon.

\section{Abbreviations}

ccRCC, clear-cell renal cell carcinoma; ChIP, chromatin immunoprecipitation; EGF, epidermal growth factor; FGF, fibroblast growth factor; HIF, hypoxiainduced factor; mTOR, mammalian target of rapamycin; RB1, retinoblastoma; VHL, von-Hippel-Lindau.

\section{Competing interests}

Research on targeted therapies in our laboratory is supported by research grants from Bayer Healthcare and 4SC.

\section{Authors' contributions}

WAS and TR researched the literature, wrote the manuscript and designed the figure.

\section{Authors' information}

WAS is research director at the Department of Urology and teaches Molecular Oncology at introductory and advanced levels. TR is a graduate student in WAS' group conducting a thesis on prostate cancer epigenetics.

\section{Acknowledgements}

We are grateful to Annemarie Koch for helpful discussions. Our current research on prostate cancer epigenetics is supported by the Deutsche Forschungsgemeinschaft and the Deutsche Krebshilfe. These funding agencies have not exerted any influence on the present article.

Published: 31 March 2011

\section{References}

1. Rubin JS, Barshishat-Kupper M, Feroze-Merzoug F, Xi ZF: Secreted WNT antagonists as tumor suppressors: pro and con. Front Biosci 2006, 11:2093-2105

2. Dalgliesh GL, Furge K, Greenman C, Chen L, Bignell G, Butler A, Davies H, Edkins S, Hardy C, Latimer C, Teague J, Andrews J, Barthorpe S, Beare D, Buck G, Campbell PJ, Forbes S, Jia M, Jones D, Knott H, Kok CY, Lau KW, Leroy C, Lin ML, McBride DJ, Maddison M, Maguire S, McLay K, Menzies A, Mironenko T, et al: Systematic sequencing of renal carcinoma reveals inactivation of histone modifying genes. Nature 2010, 463:360-363.

3. Varela I, Tarpey P, Raine K, Huang D, Ong CK, Stephens P, Davies H, Jones D, Lin ML, Teague J, Bignell G, Butler A, Cho J, Dalgliesh GL, Galappaththige D, Greenman C, Hardy C, Jia M, Latimer C, Lau KW, Marshall J, McLaren S, Menzies A, Mudie L, Stebbings L, Largaespada DA, Wessels LF, Richard S, Kahnoski RJ, Anema J, et al.: Exome sequencing identifies frequent mutation of the SWI/SNF complex gene PBRM1 in renal carcinoma. Nature 2011, 469:539-542

4. Linehan WM, Srinivasan R, Schmidt LS: The genetic basis of kidney cancer: a metabolic disease. Nat Rev Urol 2010, 7:277-285.

5. Yu J, Yu J, Mani RS, Cao Q, Brenner CJ, Cao X, Wang X, Wu L, Li J, Hu M, Gong Y, Cheng H, Laxman B, Vellaichamy A, Shankar S, Li Y, Dhanasekaran SM, Morey R, Barrette T, Lonigro RJ, Tomlins SA, Varambally S, Qin ZS, Chinnaiyan AM: An integrated network of androgen receptor, polycomb, and TMPRSS2-ERG gene fusions in prostate cancer progression. Cancer Cell 2010, 17:443-454.

6. Sorensen KD, Orntoft TF: Discovery of prostate cancer biomarkers by microarray gene expression profiling. Expert Rev Mol Diagn 2010, 10:49-64.

7. McCabe MT, Brandes JC, Vertino PM: Cancer DNA methylation: molecular mechanisms and clinical implications. Clin Cancer Res 2009, 15:3927-3937.

8. Florl AR, Schulz WA: Chromosomal instability in bladder cancer. Arch Toxicol 2008, 82:173-182. 
9. Lindgren D, Frigyesi A, Gudjonsson S, Sjödahl G, Hallden C, Chebil G, Veerla S, Ryden T, Månsson W, Liedberg F, Höglund M: Combined gene expression and genomic profiling define two intrinsic molecular subtypes of urothelial carcinoma and gene signatures for molecular grading and outcome. Cancer Res 2010, 70:3463-3472.

10. Lee JS, Leem SH, Lee SY, Kim SC, Park ES, Kim SB, Kim SK, Kim YJ, Kim WJ, Chu IS: Expression signature of E2F1 and its associated genes predict superficial to invasive progression of bladder tumors. J Clin Oncol 2010, 28:2660-2667.

doi:10.1186/gm234

Cite this article as: Schulz WA, Ribarska T: Insights into cancer mechanisms

from genomic research on urological cancers. Genome Medicine 2011, 3:20. 